\title{
Large spin splitting in the conduction band of transition metal dichalcogenide monolayers
}

\author{
K. Kośmider, J. W. González, and J. Fernández-Rossier ${ }^{\dagger}$ \\ International Iberian Nanotechnology Laboratory (INL), Av. Mestre José Veiga, 4715-330 Braga, Portugal
}

(Received 5 November 2013; published 23 December 2013)

\begin{abstract}
We study the conduction band spin splitting that arises in transition metal dichalcogenide (TMD) semiconductor monolayers such as $\mathrm{MoS}_{2}, \mathrm{MoSe}_{2}, \mathrm{WS}_{2}$, and $\mathrm{WSe}_{2}$ due to the combination of spin-orbit coupling and lack of inversion symmetry. Two types of calculation are done. First, density functional theory (DFT) calculations based on plane waves that yield large splittings, between 3 and $30 \mathrm{meV}$. Second, we derive a tight-binding model that permits to address the atomic origin of the splitting. The basis set of the model is provided by the maximally localized Wannier orbitals, obtained from the DFT calculation, and formed by 11 atomiclike orbitals corresponding to $d$ and $p$ orbitals of the transition metal (W, Mo) and chalcogenide (S, Se) atoms respectively. In the resulting Hamiltonian, we can independently change the atomic spin-orbit coupling constant of the two atomic species at the unit cell, which permits to analyze their contribution to the spin splitting at the high symmetry points. We find that-in contrast to the valence band-both atoms give comparable contributions to the conduction band splittings. Given that these materials are most often $n$-doped, our findings are important for developments in TMD spintronics.
\end{abstract}

DOI: 10.1103/PhysRevB.88.245436

PACS number(s): 73.22.-f

\section{INTRODUCTION}

Spin-orbit and exchange are the two dominant spindependent interactions in solids. Whereas the exchange splitting is only present in magnetic materials, spin-orbit coupling (SOC) is ubiquitous. The proposal of various physical effects driven by spin-orbit interactions, such as the spin Hall effect, both extrinsic ${ }^{1}$ and intrinsic, ${ }^{2,3}$ as well as the quantum spin Hall phase, ${ }^{4,5}$ together with their experimental confirmation, ${ }^{6,7}$ is opening new venues, enlarging the set of materials that could give rise to practical spintronic devices. The effects of spin-orbit interaction are particularly noticeable in materials without inversion symmetry, ${ }^{8}$ because they present spin splitting of the bands and the anomalous velocity is nonzero.

From this perspective, the new generation ${ }^{9-12}$ of semiconducting two-dimensional (2D) crystals, such as transition metal dichalgonides (TMD) monolayers (ML), is particularly appealing. The spin-orbit interaction of the constituent atoms is large and those 2D crystals have no inversion symmetry. The resulting spin splitting of the bands gives rise to the socalled spin-valley coupling, ${ }^{13}$ which has been experimentally confirmed. ${ }^{11,14-18}$ This effect is conspicuously apparent in the valence band (VB) of these materials showing the SOC splittings ranging between $150 \mathrm{meV}\left(\mathrm{MoS}_{2}\right)$ up to to $400 \mathrm{meV}$ $\left(\mathrm{WSe}_{2}\right)$. The effect of SOC in the conduction band $(\mathrm{CB})$, in contrast, have been overlooked except for a few instances. ${ }^{19-22}$ However, given that very often 2D TMD can be $n$-doped and the conduction-band spin splitting is definitely nonzero, it is of the largest interest to study this effect.

The origin of the large spin-orbit splitting of the VB is well understood: at the $K$ points, the valence-band Bloch states wave are mostly made of the metal $d$ orbitals with $\ell=2, m_{\ell}=$ $2 \tau$, where $\tau= \pm 1$ labels the valley index. ${ }^{23}$ Therefore the $m_{\ell} S_{z}$ component of SOC naturally gives a valley dependent splitting of the bands. In contrast, the dominant contribution of the CB lowest energy state comes from the $\ell=2, m_{\ell}=0$ orbitals, which cancels the spin-orbit splitting, calculated at first in perturbation theory. Thus the conduction band spin splitting was neglected in the influential seminal work of Xiao et al., ${ }^{13}$ proposing a $\mathbf{k} \cdot \mathbf{p}$ model, and most of the papers that followed. Only very recently, attention is being paid to the conduction-band splitting, ${ }^{19,24}$ using an extension of the $\mathbf{k} \cdot \mathbf{p}$ original model, ${ }^{13}$ showing that interband coupling to remote bands results in a finite $\mathrm{CB}$ splitting. However, the use of Bloch states inherent in this method obscures the atomic origin of the spin splitting, which remains to be determined and is the main focus of the present work.

In order to address the relative contributions to the $\mathrm{CB}$ splitting of the two chemical species in the unit cell, it would be convenient to describe the electronic structure of $\mathrm{MoS}_{2}$ and related 2D crystals in terms of localized atomic orbitals. However, most of the existing density functional theory $(D F T)^{25}$ calculations of the spin-orbit properties of these materials use plane waves as a basis set. ${ }^{19,20,23,26-29}$ In order to bridge the gap between plane wave and atomistic descriptions, we make use of the tight-binding (TB) Hamiltonian with a minimal basis set formed by the maximally localized Wannier functions (MLWFs). ${ }^{30}$ This approach keeps a precision of the plane-wave calculations, ${ }^{31,32}$ and at the same time allows a description of the spin-orbit coupling using the intra-atomic terms $\lambda \hat{\mathbf{L}} \cdot \hat{\mathbf{S}}$. Similar approach (we call it TB + SOC) was used to study the $\mathrm{Bi}_{2} \mathrm{Te}_{3}, \mathrm{Bi}_{2} \mathrm{Se}_{3}, \mathrm{Sb}_{2} \mathrm{Te}_{3}$ topological insulators, ${ }^{33}$ and its important advantage is that the atomic SOC of the transition metal (TM) and chalcogenide $(\mathrm{CH})$ atoms, can be varied as the $\lambda_{\mathrm{TM}}$ and $\lambda_{\mathrm{CH}}$ parameters, which permits to trace the origin of the spin splitting of the different bands. The study of spin-orbit coupling physics in other two dimensional crystals, such as graphene, using the atomic $\lambda \hat{\mathbf{L}} \cdot \hat{\mathbf{S}}$ Hamiltonian has revealed very fruitful in the past. ${ }^{34-40}$

The rest of this paper is organized as follows. In Sec. II, we present the DFT methodology and the electronic structure of the four two dimensional crystals studied here, $\mathrm{MoS}_{2}$, $\mathrm{WS}_{2}, \mathrm{MoSe}_{2}$, and $\mathrm{WSe}_{2}$. In Sec. III, we describe the way of obtaining the MLWF basis and the resulting TB Hamiltonian. In Sec. IV, we include SOC to the TB Hamiltonian as a sum of atomic terms which depend on the $\lambda_{\mathrm{TM}}$ and $\lambda_{\mathrm{CH}}$ parameters, and we determine values of these parameters. In Sec. V, we 
take advantage of the model of Sec. IV to discuss the relative contribution to the spin-orbit splitting of the conduction band of the two chemical species of the unit cell. In Sec. VI, we discuss the limitations of the model and we present our main conclusions.

\section{ELECTRONIC STRUCTURE USING DFT}

We now review the electronic properties of the $\mathrm{MoS}_{2}$, $\mathrm{MoSe}_{2}, \mathrm{WS}_{2}$, and $\mathrm{WSe}_{2} \mathrm{MLs}$ calculated with DFT in the plane wave basis as implemented in the VASP package. ${ }^{41} \mathrm{We}$ take an energy cutoff $E_{\text {cut }}=400 \mathrm{eV}$. We use the projectoraugmented waves (PAW) $)^{42,43}$ method with the $4 p, 5 s, 4 d$ valence states of the TM atom, and the $3 s, 3 p$ valence states of the $\mathrm{CH}$ atoms. The Perdew-Burke-Ernzerhof's ${ }^{44}$ version of generalized gradient approximation is used to describe the exchange correlation density functional. We use the supercell of the $1 \times 1$ periodicity and a vacuum not thinner than $17 \AA$. The Brillouin zone (BZ) is sampled with the $\Gamma$-centered $(9 \times 9 \times 1)$ Monkhorst-Pack's ${ }^{45}$ mesh of $\mathbf{k}$ points. We carry out two kinds of calculations. One with SOC included and the second one without SOC. From now on, we refer to them as to the DFT and DFT + SOC, respectively. In the DFT + SOC calculations, we use noncollinear version ${ }^{46}$ of the PAW method and SOC is described using the spherical part of the Kohn-Sham potential inside the PAW spheres. ${ }^{47}$

We briefly summarize the main features of the $\mathrm{MoS}_{2} \mathrm{ML}$ energy bands, as given by DFT and DFT + SOC [see Figs. 1(a) and 1(b), respectively]. The results for the $\mathrm{MoSe}_{2}, \mathrm{WS}_{2}$ and $\mathrm{WSe}_{2}$ MLs are very similar, and agree with the previous calculations. ${ }^{20-23,27}$ The band gap of these semiconducting MLs is direct, with the minimum of the CB and the top of the VB located at the $K$ and $K^{\prime}$ points of the BZ. All the bands at the $K$ points are spin split, but only in some instances, the splitting is so large that is appreciated by inspection in Fig. 1(b).

Analysis of the wave functions reveals that it is possible to assign a spin projection along the normal to the plane to the different Bloch states in the neighbourhood of the $K$ point. Taking advantage of this, in the following, we define the
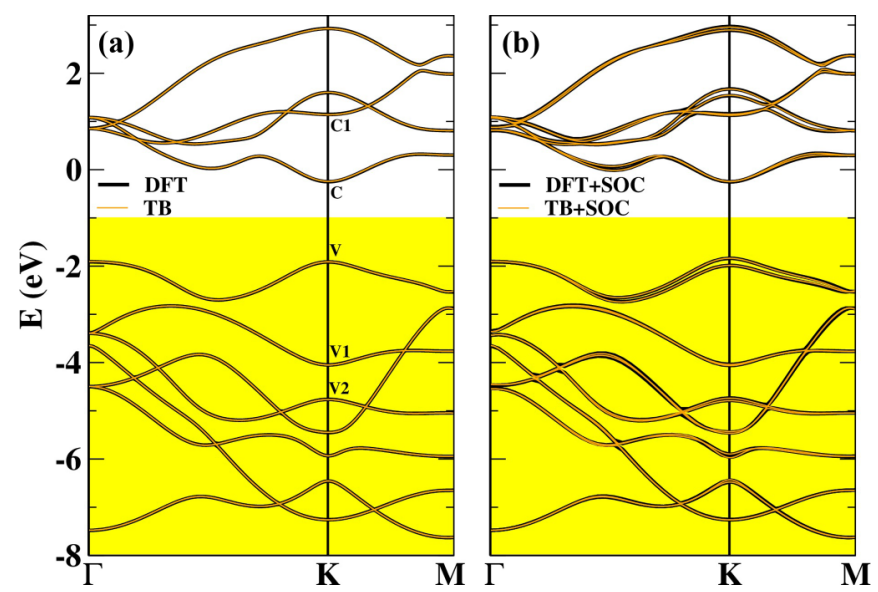

FIG. 1. (Color online) Electrons energy band structures of the $\mathrm{MoS}_{2}$ ML calculated with the DFT and TB methods: (a) bands calculated without SOC and (b) bands calculated with SOC.
TABLE I. The spin-orbit splittings $\Delta_{n}$ ( $n$ labels bands, see Fig. 1) at the $K$ point calculated for the considered TMD MLs with the $\mathrm{DFT}+\mathrm{SOC}, \mathrm{TB}+\mathrm{SOC}$, or perturbation theory (PT) methods.

\begin{tabular}{llrrrr}
\hline \hline \multirow{2}{*}{ Method } & & \multicolumn{4}{c}{$\Delta_{n}(\mathrm{meV})$} \\
\cline { 3 - 6 } DFT+ SOC & $\mathrm{C}$ & -3 & 27 & -21 & 38 \\
& $\mathrm{~V}$ & 147 & 433 & 186 & 463 \\
& $\mathrm{~V} 1$ & 24 & 70 & 27 & 88 \\
& $\mathrm{~V} 2$ & -50 & -55 & -188 & -232 \\
TB + SOC & $\mathrm{C}$ & -4 & 17 & -28 & -3 \\
& $\mathrm{~V}$ & 147 & 433 & 186 & 463 \\
& $\mathrm{~V} 1$ & 24 & 66 & 29 & 64 \\
& $\mathrm{~V} 2$ & -50 & -55 & -188 & -232 \\
PT & $\mathrm{C}$ & -1 & 13 & -11 & 7 \\
\hline \hline
\end{tabular}

splitting of a energy band $n$ with momentum $\mathbf{k}$ as

$$
\Delta_{n}(\mathbf{k}) \equiv \epsilon_{n \uparrow}(\mathbf{k})-\epsilon_{n \downarrow}(\mathbf{k}) .
$$

With this convention, the splitting can be either positive or negative. Time-reversal symmetry warrants that $\Delta_{n}(\mathbf{k})=$ $-\Delta_{n}(-\mathbf{k})$, which implies that spin splittings have opposite signs in $K$ and $K^{\prime}$ valleys. ${ }^{13}$ The spin splittings of the relevant bands at the $K$ point are listed in Table I. The spin-orbit splitting at the top of the VB, range between $147 \mathrm{meV}$ for $\mathrm{MoS}_{2}$ and $463 \mathrm{meV}$ for $\mathrm{WSe}_{2}$. The same splittings for the CB vary from $-3 \mathrm{meV}$ for $\mathrm{MoS}_{2}$ to $38 \mathrm{meV}$ for $\mathrm{WSe}_{2}$. They are smaller than those of the VB, but definitely large enough as to be observed. It is worth noticing that only in the case of the conduction band, the sign of $\Delta$ is not the same for all the compounds, for reasons explained below.

We now discuss the population analysis of the DFT Bloch states. This sheds some light on the origin of their spin splittings. Both VB and CB are predominantly made of the TM atom $d(\ell=2)$ orbitals and a smaller but not negligible contribution coming from the $p\left(\ell=1, m_{\ell}= \pm 1\right)$ orbitals of the chalcogen atoms. The main difference between VB and $\mathrm{CB}$ bands lies in the $m_{\ell}$ number of $d$ orbitals, which is equal \pm 2 in the $\mathrm{VB}$ and 0 in the $\mathrm{CB}$. This picture is in agreement with earlier work. ${ }^{13,19-23}$ In the discussion below we shall also make use the fact that the Bloch state labeled as V2 at the $K$ point is made exclusively of the chalcogen $p$ orbitals $(\ell=1$, $m_{\ell}=+1$ ), without mixing to the metal $d$ orbitals.

\section{MAXIMALLY LOCALIZED WANNIER FUNCTIONS BASIS}

The Wannier functions ${ }^{48}$ (WF) permit to define a localized basis set by performing a unitary transformation over a set of Bloch states that diagonalize the DFT Hamiltonian. Although there is not a unique way of doing such a wannierization, we adopt the method criteria of maximal localization ${ }^{30}$ and we use the WANNIER $90^{49}$ code to find the basis of MLWFs. This approach has already been used for $\mathrm{MoS}_{2}$ and related transition metal dichalcogenides before ${ }^{50}$ obtaining results in line with those discussed here. In our case, the set is formed by the group of 11 bands distributed around the band gap, as shown in Fig. 1(a). 
(a)

(b)

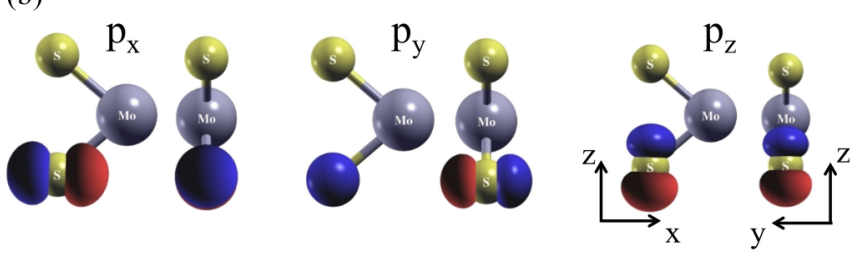

(c)

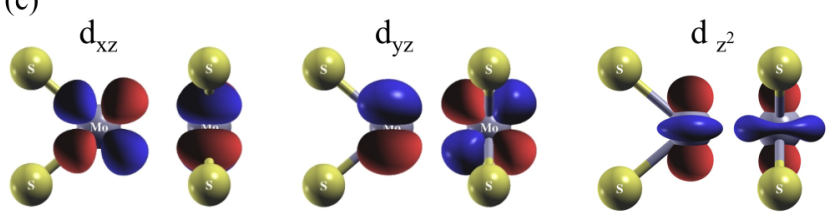

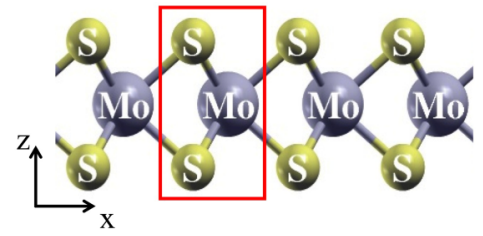
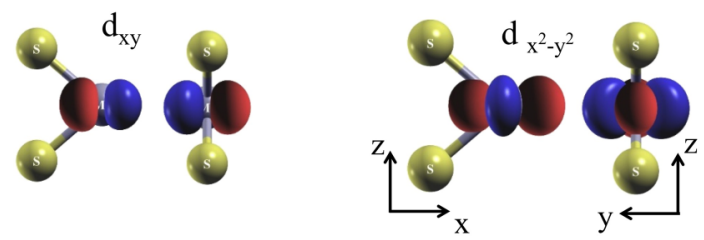

FIG. 2. (Color online) The MLWF basis used to express TB Hamiltonian (2) of the $\mathrm{MoS}_{2}$ ML: (a) Side view of the monolayer, (b) and (c) contour-surface plot of the three $p$ orbitals of sulfur and the five $d$ orbitals of molybdenum. Figure prepared with XCRYSDEN. ${ }^{51}$

The first step of the procedure consist of the projection of the Bloch states $\left|\psi_{\mathbf{k}, n}\right\rangle$ over certain a set of localized functions, which in this case, are taken as the $p$ and $d$ atomic orbitals of the chalcogenide and metallic atom, respectively, motivated by the population analysis discussed above. Importantly, in the case of 2D TMDC, the MLWF are centered around the atoms, their localization radius is smaller than the interatomic distance and, in the neighborhood of the atoms, they have the symmetry of the real spherical harmonics. A numerical measure of the localization is given by the localization functional $^{30} \Omega$. In our case, after 100 iterative steps, we obtain a total spread 18.23/20.85/20.28/23.25 $\AA^{2}$, summing over the 11 Wannier orbitals, for $\mathrm{MoS}_{2} / \mathrm{MoSe}_{2} / \mathrm{WS}_{2} / \mathrm{WSe}_{2}$, which yields an average size per Wannier orbital of 1.29/1.38/1.36/1.45 $\AA$.

The isosurfaces of the MLWF obtained for $\mathrm{MoS}_{2}$ are presented in Fig. 2. Their real spherical harmonic symmetry is apparent. In the following, we label the MLWF as $|\mathbf{R} O\rangle$, where $\mathbf{R}$ defines a unit cell inside the crystal and $O$ refers to one the 11 atomiclike MLWF inside the unit cell. We refer to them using their real spherical harmonic symmetry, as shown in Fig. 2. However, the shape (not shown) of the tails of the MLWF is different from that of the core. Therefore MLWFs are not identical to atomic orbitals for which the angular symmetry is independent of the distance to the nuclei.

\section{A. Wannier Hamiltonian}

The wannierization procedure yields the basis of 11 atomiclike orbitals $|\mathbf{R} O\rangle$, and-more importantly-a faithful representation of the DFT Hamiltonian in that basis. Thus, for a given pair of the atomiclike MLWF orbitals $O$ and $O^{\prime}$, located in unit cells $\mathbf{R}$ and $\mathbf{R}^{\prime}$, we obtain the representation of the DFT Hamiltonian $\left\langle\mathbf{R} O|\hat{H}| \mathbf{R}^{\prime} O^{\prime}\right\rangle$. Taking advantage of the Bloch theorem, the Hamiltonian for the entire crystal can be block diagonalized in the usual way, resulting in the following wave-vector-dependent Hamiltonian matrix:

$$
\mathcal{H}_{O O^{\prime}}(\mathbf{k})=\sum_{\mathbf{R}} e^{i \mathbf{k} \cdot \mathbf{R}}\left\langle\mathbf{0} O\left|\hat{H}_{\mathrm{DFT}}\right| \mathbf{R} O^{\prime}\right\rangle,
$$

where the sum runs over all the unit cells of the crystal, labeled with $\mathbf{R}$. In practice, the localized nature of the MLWFs permits to truncate the sum down to a few neighbors. In the following, we shall denote by $\hat{\mathcal{H}}_{0}(\mathbf{k})$ the Hamiltonian operator associated to the matrix of Eq. (2). Importantly, the dimension of the matrix (2) is as small as the size of the MLWF basis (11 in the present case), which makes the numeric diagonalization computationally inexpensive. The resulting energy bands are-not surprisingly (given their formal equivalence)-very similar to those obtained from DFT as shown in Fig. 1(a). Minor differences (not appreciated at the energy scale used in the figure) arise from the truncation in the number of bands, i.e., due to interband coupling to remote high- and low-energy bands that have been excluded in the Wannier Hamiltonian but are present in the DFT calculation.

The eigenstates of Hamiltonian (2) are a linear combination of the MLWFs which-as discussed above-have real spherical harmonic symmetry close to the atom cores. In order to understand the spin splittings, it is convenient to define a new basis of orbitals localized around atom $A$, denoted by $\left|A_{m_{\ell}}^{\ell}\right\rangle$, which has the symmetry of the eigenstates of the atomic angular momentum operator. In other words, we move from a real basis to the usual spherical harmonics with well defined $m_{\ell}$. In the rest of this paper, we use the following notation to relate the $n$th band Bloch state at the $K$ point with the atomically localized orbitals $\left|A_{m_{\ell}}^{\ell}\right\rangle$ :

$$
\left|\psi_{K, n}\right\rangle=\alpha_{n}\left|\mathrm{TM}_{m_{\ell}}^{\ell=2}\right\rangle+\beta_{n}\left(\left|\mathrm{CH}_{m_{\ell}}^{\ell=1}\right\rangle+s_{n}\left|\mathrm{CH}_{m_{\ell}}^{\ell=1}\right\rangle\right),
$$

where $\alpha_{n}$ and $\beta_{n}$ are coefficients, and $s_{n}= \pm 1(+1$ for the bands $\mathrm{C}, \mathrm{V}, \mathrm{V} 1$ and -1 for $\mathrm{C} 1$ and $\mathrm{V} 2$ ).

Importantly, since the MLWFs do not rigorously have spherical harmonic symmetry, the $\left|A_{m_{\ell}}^{\ell}\right\rangle$ are not rigorously eigenstates of the atomic angular momentum operator. However, in the rest of this work, we adopt the approximation that the $\left|A_{m_{\ell}}^{\ell}\right\rangle$ are indeed eigenstates of the atomic orbital angular momentum operator. The validity of this approach is justified by the fairly good agreement with the DFT results, discussed below.

In Table II, we show $\left|\alpha_{n}\right|^{2}$ and $\left|\beta_{n}\right|^{2}$. It is apparent that the $\mathrm{CB}$ and VB are mostly made of the transition metal $d$ orbitals, with $m_{\ell}$ equal 0 and 2 , respectively. The small variations of the coefficient squares $\alpha^{2}$ and $\beta^{2}$ along the different materials inform of their similar electronic structure. It must be noticed that the contributions of the orbitals localized on the $\mathrm{CH}$ atoms, given by $2 \beta_{n}^{2}$, are larger than $10 \%$, and thereby they can account for a fraction of the spin splitting, as it actually happens. Inspection of the wave functions also reveals their odd/even character with respect to reflection across the $z=0$ plane. Specifically, the wave functions of bands $\mathrm{C}$ and $\mathrm{V}$ are 
TABLE II. Table of projections of Bloch states at $K$ over the $\left|A_{m_{\ell}}^{\ell}\right\rangle$ basis [see Eq. (3)]. The leftmost columns denote the $m_{\ell}$ relevant for each band. Since there are two equivalent chalcogen atoms per unit cell, the normalization criterion is $\left|\alpha_{n}\right|^{2}+2\left|\beta_{n}\right|^{2}=1$.

\begin{tabular}{|c|c|c|c|c|c|c|c|c|c|c|}
\hline \multirow[b]{2}{*}{$n$} & \multicolumn{2}{|c|}{$m_{\ell}$} & \multicolumn{2}{|c|}{$\mathrm{MoS}_{2}$} & \multicolumn{2}{|c|}{$\mathrm{WS}_{2}$} & \multicolumn{2}{|c|}{$\mathrm{MoSe}_{2}$} & \multicolumn{2}{|c|}{$\mathrm{WSe}_{2}$} \\
\hline & $|\mathrm{TM}\rangle$ & $|\mathrm{CH}\rangle$ & $\alpha_{n}^{2}$ & $\beta_{n}^{2}$ & $\alpha_{n}^{2}$ & $\beta_{n}^{2}$ & $\alpha_{n}^{2}$ & $\beta_{n}^{2}$ & $\alpha_{n}^{2}$ & $\beta_{n}^{2}$ \\
\hline $\mathrm{C} 1$ & -1 & +1 & 0.63 & 0.19 & 0.63 & 0.19 & 0.65 & 0.18 & 0.65 & 0.18 \\
\hline $\mathrm{C}$ & 0 & -1 & 0.86 & 0.07 & 0.90 & 0.05 & 0.86 & 0.07 & 0.89 & 0.05 \\
\hline V & +2 & +1 & 0.80 & 0.10 & 0.79 & 0.11 & 0.82 & 0.09 & 0.79 & 0.10 \\
\hline V1 & +1 & 0 & 0.28 & 0.36 & 0.25 & 0.38 & 0.34 & 0.33 & 0.30 & 0.35 \\
\hline V2 & $\ldots$ & -1 & $\ldots$ & 0.5 & $\ldots$ & 0.5 & $\ldots$ & 0.5 & $\ldots$ & 0.5 \\
\hline
\end{tabular}

even and those of bands $\mathrm{C} 1, \mathrm{~V} 1$, and $\mathrm{V} 2$ are odd, in agreement with previous results. ${ }^{19}$

\section{ATOMIC SOC}

The Wannier Hamiltonian just described is derived from a DFT calculation where SOC has been deliberately excluded. We now proceed to add the atomic spin-orbit coupling into the TB Hamiltonian

$$
\hat{\mathcal{V}}_{\mathrm{SOC}}=\sum_{A} \lambda_{A} \hat{\mathbf{L}}_{A} \cdot \hat{\mathbf{S}}
$$

where $\lambda_{A}$ is a scalar that measure the strength of the atomic $\mathrm{SOC}, \hat{\mathbf{L}}_{A}$ is the angular momentum operator acting on an atom $A$, and $\hat{\mathbf{S}}$ are the spin $1 / 2$ Pauli matrices operators. As discussed after Eq. (3), we assume that

$$
\begin{aligned}
\left\langle A_{m_{\ell}}^{\ell}\left|\hat{L}_{A}^{ \pm}\right| A_{m_{\ell}^{\prime}}^{\ell}\right\rangle & =\sqrt{\ell(\ell+1)-m_{\ell}\left(m_{\ell}^{\prime} \pm 1\right)} \delta_{m_{\ell}, m_{\ell}^{\prime}+1}, \\
\left\langle A_{m_{\ell}}^{\ell}\left|\hat{L}_{A}^{z}\right| A_{m_{\ell}^{\prime}}^{\ell}\right\rangle & =m_{\ell} \delta_{m_{\ell}, m_{\ell}^{\prime}} .
\end{aligned}
$$

The addition of $\hat{\mathcal{V}}_{\text {soc }}$ to $\hat{\mathcal{H}}_{0}(\mathbf{k})$-the TB operator computed without SOC-leads to the following TB Hamiltonian:

$$
\hat{\mathcal{H}}(\mathbf{k})=\hat{\mathcal{H}}_{0}(\mathbf{k})+\hat{\mathcal{V}}_{\text {SOC }},
$$

which is the main result of this work. The presence of $\hat{\mathcal{V}}_{\text {SOC }}$ in Eq. (6) causes spin splittings $\Delta_{n}$, which depend on two parameters $\lambda_{\mathrm{CH}}$ and $\lambda_{\mathrm{TM}}$.

We are now in position of achieving two goals. First, we can verify the validity of our approach fitting the $\lambda$ parameters that give a best agreement between the bands of Hamiltonian (6) and those obtained with the DFT + SOC method, paying special attention to the spin splittings $\Delta_{n}$ close to the $K$ point. Second, we can determine the contribution each atom to the spin-orbit splitting a various bands, with an attention to the conduction band.

\section{A. Perturbative estimate of $\lambda$}

It is very instructive to obtain formal expressions for the $\Delta_{n}$ splittings treating $\hat{\mathcal{V}}_{\text {SOC }}$ to first order in perturbation theory. A comparison of these expressions with the values calculated using DFT + SOC method yields a first estimate for $\lambda_{\mathrm{CH}}$ and $\lambda_{\mathrm{TM}}$. Choosing $\hat{z}$ as the spin quantization axis, the shift of the
TABLE III. The atomic SOC parameters $\lambda_{\mathrm{TM}}$ and $\lambda_{\mathrm{CH}}$ of the considered TMD MLs. Comparison of the values estimated with

\begin{tabular}{|c|c|c|c|c|}
\hline & \multicolumn{2}{|c|}{$\lambda_{\mathrm{TM}}(\mathrm{meV})$} & \multicolumn{2}{|c|}{$\lambda_{\mathrm{CH}}(\mathrm{meV})$} \\
\hline & PT & $\mathrm{TB}+\mathrm{SOC}$ & PT & $\mathrm{TB}+\mathrm{SOC}$ \\
\hline $\mathrm{MoS}_{2}$ & 87 & 86 & 50 & 52 \\
\hline $\mathrm{WS}_{2}$ & 274 & 271 & 55 & 57 \\
\hline $\mathrm{MoSe}_{2}$ & 94 & 89 & 188 & 256 \\
\hline $\mathrm{WSe}_{2}$ & 261 & 251 & 232 & 439 \\
\hline
\end{tabular}
perturbation theory (PT) with the values calculated by fitting the $\Delta_{\mathrm{V}}$ and $\Delta_{\mathrm{V} 2}$ splittings to the values obtained from DFT + SOC method (see Table I).

levels with spin $\sigma$, to first order in perturbation theory, reads

$$
\delta \epsilon_{n \sigma}(\mathbf{k})=\frac{\sigma}{2}\left\langle\psi_{n \mathbf{k}}\left|\sum_{A} \lambda_{A} \hat{L}_{A}^{z}\right| \psi_{n \mathbf{k}}\right\rangle .
$$

Since there are two unknowns, we implement this procedure with two bands, $\left|\psi_{\mathrm{v} 2, K}\right\rangle$ and $\left|\psi_{\mathrm{v}, K}\right\rangle$ at the $K$ point. In the case of $\mathrm{V} 2$ the contribution from the TM is strictly null, so that first-order perturbation theory yields

$$
\Delta_{\mathrm{v} 2}=\left\langle\psi_{\mathrm{v} 2, K}\left|\sum_{A} \lambda_{A} \hat{L}_{A}^{z}\right| \psi_{\mathrm{v} 2, K}\right\rangle=-\lambda_{\mathrm{CH}},
$$

which permits to relate directly the splitting of the V2 band at the $K$ point with the chalcogenide spin-orbit coupling. In the case of the VB, the first-order perturbation theory yields

$$
\Delta_{\mathrm{V}}=2 \alpha_{\mathrm{C}}^{2} \lambda_{\mathrm{TM}}+2 \beta_{\mathrm{C}}^{2} \lambda_{\mathrm{CH}} .
$$

Combining these two equations, we obtain an estimate for $\lambda_{\mathrm{TM}}$ and $\lambda_{\mathrm{CH}}$, shown in the PT columns of Table III, together with the estimates using a nonperturbative fitting described below.

The first point to notice is that across different materials (except in the case of Se) the values of $\lambda$ undergo variations smaller than $10 \%$. This is in line with the general notion that for a given atom, spin-orbit coupling does not vary much from compound to compound. These small variations are a first indication of the validity of our methodology. The second point is that these values are in line with those reported for neutral $\mathrm{S} / \mathrm{Se}$ atoms $(50 / 220 \mathrm{meV})^{52}$ as well as for Mo $(78 \mathrm{meV}) .^{53}$ In order to understand why $\lambda_{\mathrm{Se}}>\lambda_{\mathrm{Mo}}$ in spite of the fact that $Z_{\mathrm{Se}}<Z_{\mathrm{Mo}}$, we recall that orbitals with larger $n$ and/or larger $\ell$ have a smaller overlap with the electric field of the nucleus and, thereby, reduced spin-orbit coupling. This concept is illustrated by the hydrogen model ${ }^{53}$ in which $\lambda \propto\left(Z^{4}\right) /\left[n^{3} \ell(\ell+1)(\ell+\right.$ $1 / 2)$ ], leading to $\lambda_{\mathrm{Se}} \gg \lambda_{\mathrm{Mo}}$.

Moreover, it must be kept in mind that the localization of Wannier and atomic orbitals can be different. Thereby, a scaling of the $\lambda$ for the Wannier orbitals, compared to the atomic orbitals, is expected. Our calculations indicate that this is not a large effect, endorsing the notion that the MLWF used in our calculation are similar to the atomic orbitals.

\section{B. Determination of $\lambda$ with Wannier Hamiltonian}

We now discuss a second and more accurate way to determine the $\lambda_{\mathrm{TM}}$ and $\lambda_{\mathrm{CH}}$ parameters. For a given value 


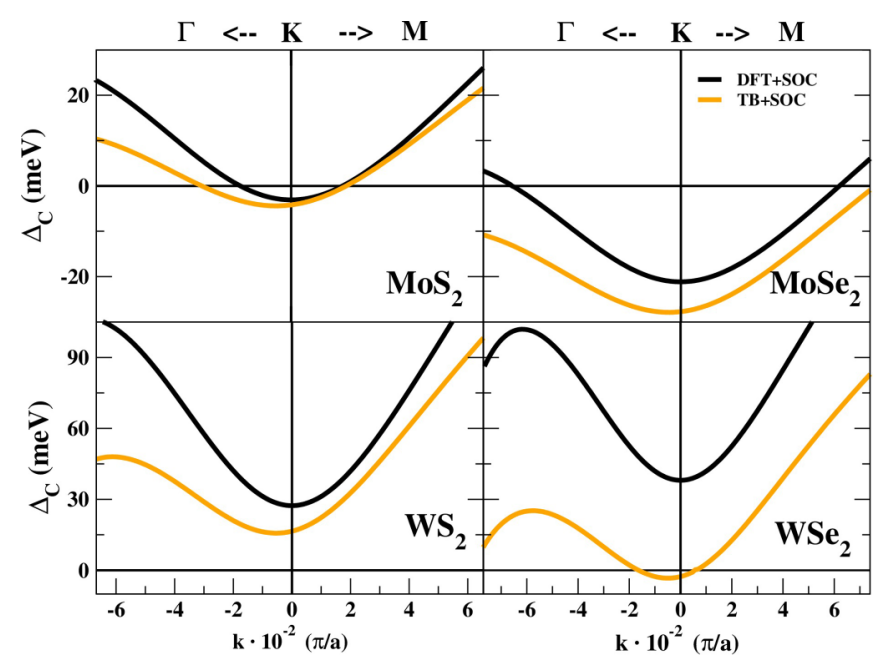

FIG. 3. (Color online) Comparison of spin-orbit splitting of the conduction band around the $K$ point calculated with the TB + SOC and DFT + SOC methods.

of $\lambda_{\mathrm{TM}}$ and $\lambda_{\mathrm{CH}}$, numerical diagonalization of the Hamiltonian in Eq. (6) yields a set of spin-split bands.

As in the perturbative case, we determine $\lambda$ 's by fitting the spin splitting at the $K$ point of both valence and V2 bands to those obtained in the DFT + SOC calculations. The values of $\lambda_{\mathrm{TM}}$ and $\lambda_{\mathrm{CH}}$ parameters estimated this way are listed in the TB + SOC columns of Table III. They are close to the PT values except the $\lambda_{\mathrm{Se}}$ in the $\mathrm{WSe}_{2}$ ML. Possible explanations for this are detailed below.

In Fig. 1, we show a comparison of the DFT + SOC bands (left panel) and with the just described TB + SOC method (right panel). It should be noted that, fixing the splitting of two bands at the $K$ point, yields a fairly good agreement for all the bands on the entire Brillouin zone, giving additional support to the methodology.

A more quantitative comparison between the $\mathrm{TB}+\mathrm{SOC}$ and the DFT + SOC calculations is shown in Table I where we compare the spin splitting of several bands at the $K$ point obtained with the two methods. Of course, by construction of the method, the agreement for the VB and V2 is perfect. In addition, it is apparent that the $\mathrm{TB}+\mathrm{SOC}$ provides a fairly good quantitative agreement for the spin splitting of the conduction and $\mathrm{V} 1$ bands, except for $\mathrm{WSe}_{2}$. In Fig. 3, we compare $\Delta_{\mathrm{C}}(\mathbf{k})$ for DFT + SOC and TB + SOC along the $\Gamma-K-M$ high symmetry points. It is apparent that the TB method captures the nontrivial momentum dependence featured by the DFT + SOC, although there is a systematic off-set which is also larger for $\mathrm{WSe}_{2}$.

\section{CONDUCTION BAND SPIN-ORBIT SPLITTING}

We are now in a position to discuss the mechanism for the conduction band splitting in the TMD monolayers. Inspection of the $m_{\ell}$ values in Table II reveals that $\Delta_{\mathrm{C}}$ should vanish to first order in $\lambda_{\mathrm{TM}}$, and have a small linear contribution in $\lambda_{\mathrm{CH}}$. To check this out, we plot in Fig. 4 the $\Delta_{\mathrm{C}}(K)$ splitting, keeping one of the $\lambda$ values as given in table III (TB + SOC values), and varying the other. The $\Delta_{\mathrm{C}}\left(\lambda_{\mathrm{CH}}\right)$ dependence (for $\lambda_{\mathrm{TM}}=\mathrm{const}$ ) is a straight line with negative slope. This can

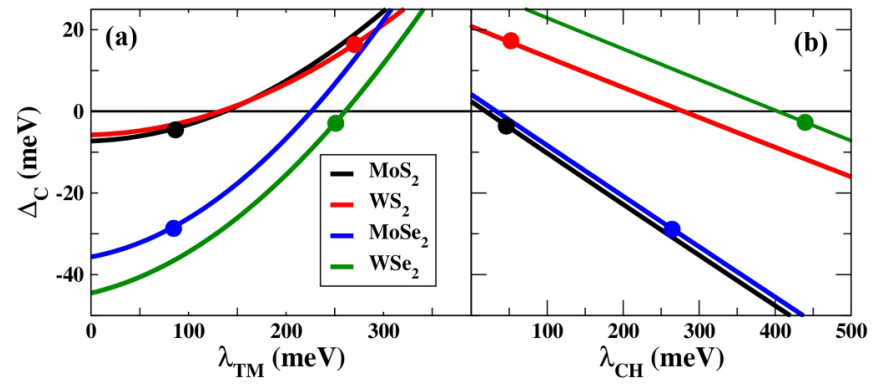

FIG. 4. (Color online) Spin-orbit splitting of the conduction band at the $K$ point calculate for the considered TMD MLs as a function of $\lambda_{\mathrm{TM}}$ (a) and $\lambda_{\mathrm{CH}}$ (b) parameters. The corresponding $\lambda_{\mathrm{CH}}$ (a) and $\lambda_{\mathrm{TM}}$ (b) parameters are fixed to the values estimated with the TB + SOC method (see Table I). Bold dots mark the $\lambda_{\mathrm{TM}}$ and $\lambda_{\mathrm{CH}}$ parameters calculated with the same method.

be understood within first-order perturbation theory, which yields the following expression for the chalcogen atom SOC contribution to the splitting:

$$
\delta \epsilon_{\mathrm{C} \uparrow}^{(1)}(K)-\delta \epsilon_{\mathrm{C} \downarrow}^{(1)}(K)=\left\langle\psi_{K, \mathrm{C}}\left|\lambda_{\mathrm{CH}} \hat{L}_{\mathrm{CH}}^{z}\right| \psi_{K, \mathrm{C}}\right\rangle=-\lambda_{\mathrm{CH}} \beta_{\mathrm{C}}^{2},
$$

where the negative sign comes from the fact that, at the $K$ point, the CB Bloch state overlaps with the $m_{\ell}=-1$ chalcogenide atomiclike state (see Table II). With this equation, the negative slopes $\partial \Delta_{\mathrm{C}} / \partial \lambda_{\mathrm{CH}}$ in Fig. 4(b) became clear. They are controlled by $\beta_{\mathrm{C}}$ (see Table II) and are the same for the tungsten based $\mathrm{WS}_{2}$ and $\mathrm{WSe}_{2}$ compounds as well as molybdenum based $\mathrm{MoS}_{2}$ and $\mathrm{MoSe}_{2}$ compounds.

In contrast, the $\Delta_{\mathrm{C}}\left(\lambda_{\mathrm{TM}}\right)$ dependence (for $\lambda_{\mathrm{CH}}=$ const) is not linear-reflecting the inter band character of this contribution-and has a positive sign. Of course, opposite signs and trends are found at the $K^{\prime}$ point, on account of time reversal symmetry. The well defined sign of the interband contribution to the $\mathrm{CB}$ spin splitting is understood as follows. First, we use second-order perturbation theory, that yields positive (negative) shifts via interband coupling to states below (above) in energy. Second, given the fact that at the $K$ point the Bloch states overlap with states with a well defined handedness, together with the angular momentum conservation, result in a spin-selective interband coupling. Thus the TM SOC can connect the CB state $\left(m_{\ell}=-1\right)$ with spin $\uparrow$ only to the states with the opposite values of $m_{\ell}(+1)$ and spin $(\downarrow)$, which happen to be available at the band V1, providing a positive contribution of the shift given by

$$
\delta \epsilon_{\mathrm{c} \uparrow}^{(2)}(K)=\frac{1}{4} \frac{\lambda_{\mathrm{TM}}^{2}\left|\left\langle\psi_{\mathrm{C}, K}\left|\hat{L}^{-}\right| \psi_{\mathrm{v} 1, K}\right\rangle\left\langle\uparrow\left|\hat{S}^{+}\right| \downarrow\right\rangle\right|^{2}}{\epsilon_{\mathrm{c}}(K)-\epsilon_{\mathrm{v} 1}(K)},
$$

whereas the coupling of the $\mathrm{CB}$ to $\mathrm{V} 1$ give a null shift of $\delta \epsilon_{\mathrm{C} \downarrow}^{(2)}(K)$. In contrast, the $\mathrm{CB}$ state with spin $\downarrow$, can only connect to states with $m_{\ell}=-1$, , which happen to be available at the $\mathrm{C} 1$ state, giving a negative shift to the $\downarrow$ level and thereby another positive contribution to the splitting:

$$
\delta \epsilon_{\mathrm{C} \downarrow}^{(2)}(K)=\frac{1}{4} \frac{\lambda_{\mathrm{TM}}^{2}\left|\left\langle\psi_{\mathrm{C}, K}\left|\hat{L}^{+}\right| \psi_{\mathrm{Cl}, K}\right\rangle\left\langle\downarrow\left|\hat{S}^{-}\right| \uparrow\right\rangle\right|^{2}}{\epsilon_{\mathrm{C}}(K)-\epsilon_{\mathrm{Cl}}(K)} .
$$

We now define $\delta_{\mathrm{C}, \mathrm{V} 1} \equiv \epsilon_{\mathrm{C}}(K)-\epsilon_{\mathrm{V} 1}(K)$ and $\delta_{\mathrm{C}, \mathrm{C} 1} \equiv$ $\epsilon_{\mathrm{C}}(K)-\epsilon_{\mathrm{C} 1}(K)$. Combining Eqs. (10)-(12) with Eq. (3), 
using $\left|\left\langle\uparrow\left|\hat{S}^{+}\right| \downarrow\right\rangle\right|^{2}=1$ and $\left|\left\langle\psi_{\mathrm{C}, K}\left|\hat{L}^{-}\right| \psi_{n, K}\right\rangle\right|^{2}=2 \alpha_{\mathrm{C}}^{2} \alpha_{n}^{2}$, with $n=\mathrm{C} 1$, V1, we can write the following perturbative expression for the $\mathrm{CB}$ spin splitting at the $K$ valley:

$$
\Delta_{\mathrm{C}}(K)=-\lambda_{\mathrm{CH}} \beta_{\mathrm{C}}^{2}+\frac{\left(\lambda_{\mathrm{TM}} \alpha_{\mathrm{C}}\right)^{2}}{2}\left(\frac{\alpha_{\mathrm{v} 1}^{2}}{\delta_{\mathrm{C}, \mathrm{V} 1}}-\frac{\alpha_{\mathrm{C} 1}^{2}}{\delta_{\mathrm{C}, \mathrm{C} 1}}\right) .
$$

However, since $\delta_{\mathrm{C}, \mathrm{V} 1}>0 \mathrm{eV}$ and $\delta_{\mathrm{C}, \mathrm{C} 1}<0 \mathrm{eV}$, the two terms proportional to $\lambda_{\mathrm{TM}}^{2}$ are positive. The values of $\Delta_{\mathrm{C}}(K)$ calculated with Eq. (13) for the four TMD MLs are listed in Table I (see row PT). It is apparent that the perturbative calculation captures the trend of the nonperturbative results calculation result and provides a qualitative insight of the contribution of each atom to the conduction band splitting.

In summary, the $\mathrm{CB}$ splitting has two contributions with opposite signs. For the $K$ valley, the chalcogen SOC gives a negative contribution and the transition metal a positive one. This explains the material dependent sign. Thus $\mathrm{WS}_{2}$ combines the largest positive with the smallest negative contribution, resulting in a clearly positive splitting. On the opposite side, $\mathrm{MoSe}_{2}$ combines the smaller TM SOC and the largest $\mathrm{CH}$ SOC, resulting in the largest negative contribution. In $\mathrm{MoS}_{2}$, the two competing contributions are the smallest (comparing to the other considered MLs) and go a long way to cancel each other: sulfur alone would give $\Delta \simeq-3 \mathrm{meV}$, whereas Mo alone would give $\Delta \simeq+2 \mathrm{meV}$. It must be noted that previous work by Liu et al. ${ }^{54}$ has a similar discussion using perturbation theory but considering only contributions from the transition metal atoms and dropping those coming from the chalcogens. As a result, their perturbative estimates for the conduction band spin splittings come out always with the same sign for all materials, in contrast with DFT. This points out the relevance of the chalcogen spin-orbit coupling, specifically for the $\mathrm{MoS}_{2}$ and $\mathrm{MoSe}_{2}$, for which the relative contribution coming from the chalcogen is larger.

\section{DISCUSSION AND CONCLUSIONS}

We now discuss some of the limitations of our model. First, it is apparent that the agreement between the TB + SOC model and the DFT $+\mathrm{SOC}$ results is not good in the case $\mathrm{WSe}_{2}$. This is reflected in the discrepancy of the CB spin splitting shown in Table I and in the large variations of the value of $\lambda_{\text {Se }}$ determined using perturbation theory and the nonpeturbative method (see Table III). This is due in part to the truncation in the number of bands in the TB method. Interband contributions to bands omitted in the TB model contribute to the spin-orbit splitting, and this effect is of course larger for $\mathrm{WSe}_{2}$ for which both $\lambda$ 's are largest.

A second contribution to this discrepancy might arise from the fact that the MLWF are not exactly the same than atomic orbitals. However, the differences are large only in the interstitial region and should weakly affect the spin-orbit physics. In contrast, the loss of atomic symmetry in the interstitial region clearly explains why our attempts, not discussed above, to parametrize the Wannier-TB Hamiltonian with a Slater Koster ${ }^{55}$ parameters have failed. Therefore the method discussed in this paper needs to be modified in order to map the DFT calculation into a TB model parametrized with a few Slater Koster parameters, in the line of recent work. ${ }^{54,56,57}$ A third missing ingredient in the $\mathrm{TB}+\mathrm{SOC}$, compared to the DFT + SOC, are interatomic terms, as opposed to the intra-atomic contributions described in Eq. (4).

In summary, DFT calculations show that semiconducting two dimensional transition metal dichalcogenides have spinorbit splittings at the conduction band that-although smaller than those at the valence band-are definitely large enough to be relevant experimentally. ${ }^{58}$ In order to understand the chemical origin of the splitting, we have derived a tight-binding Hamiltonian (6) using the maximally localized Wannier functions as a basis. Taking advantage of their atomiclike character, it is possible to add the atomic spin-orbit coupling operators to the tight-binding model, using the atomic $\lambda$ as adjustable parameters. We have found that this method describes very well the bands in the energy range from -8 to $3 \mathrm{eV}$ around the Fermi level. The tight-binding model permits to determine that both types of atoms, metal and chalcogen, contribute to the conduction band spin splitting with opposite signs. This naturally explains why conduction band spin-orbit splittings of the $\mathrm{WS}_{2}$ and $\mathrm{MoSe}_{2}$ present opposite signs.

Our findings have implications on a wide array of spin related physical phenomena that are being explored in two dimensional transition metal dichalcogenides and their nanostructures, ${ }^{59}$ including the conduction band Landau Levels, ${ }^{60}$ spin relaxation, ${ }^{61}$ exciton spin selection rules, ${ }^{62}$ RKKY coupling, ${ }^{63}$ as well as the spin and valley Hall effects. ${ }^{13,50,64}$

Note added. Recently, related work has been posted, see Ref. 65.

\section{ACKNOWLEDGMENTS}

We acknowledge fruitful discussions with A. Kormányos, J. Jung, A. H. MacDonald, I. Souza, J. L. Martins, E. V. Castro, and J. L. Lado. We thankfully acknowledge the computer resources, technical expertise and assistance provided by the Red Española de Supercomputación. JFR acknowledges financial supported by MEC-Spain (FIS2010-21883-C02-01) and Generalitat Valenciana (ACOMP/2010/070), Prometeo. This work has been financially supported in part by FEDER funds. We acknowledge financial support by Marie-Curie-ITN 607904-SPINOGRAPH.

\footnotetext{
* Current address: Centro de Física de Materiales (CSIC-UPV/EHU)Material Physics Center (MPC), Paseo Manuel de Lardizábal 5, 20018, San Sebastián, Spain.

${ }^{\dagger}$ On leave from Departamento de Física Aplicada, Universidad de Alicante, Spain.
}

${ }^{1}$ J. E. Hirsch, Phys. Rev. Lett. 83, 1834 (1999).

${ }^{2}$ S. Murakami, N. Nagaoisa, and S. C. Zhang, Science 301, 1348 (2003).

${ }^{3}$ N. Nagaosa, J. Sinova, S. Onoda, A. H. MacDonald, and N. P. Ong, Rev. Mod. Phys. 82, 1539 (2010). 
${ }^{4}$ C. L. Kane and E. J. Mele, Phys. Rev. Lett. 95, 146802 (2005); 95, 226801 (2005).

${ }^{5}$ B. A. Bernevig, T. L. Hughes, S. C: Zhang, Science 314, 1757 (2006).

${ }^{6}$ S. O. Valenzuela and M. Tinkham, Nature (London) 442, 176 (2006).

${ }^{7}$ M. Konig, S. Wiedmann, C. Brune, A. Roth, H. Buhmann, L. W. Molenkamp, X. L. Qi, and S. C. Zhang, Science 318, 5851 (2007). ${ }^{8}$ D. Xiao, M.-C. Chang, and Q. Niu, Rev. Mod. Phys. 82, 1959 (2010).

${ }^{9}$ Q. H. Wang, K. Kalantar-Zadeh, A. Kis, J. N. Coleman, and M. S. Strano, Nat. Nanotechnol. 7, 699 (2012).

${ }^{10}$ M. Chhowalla, H. S. Shin, G. Eda, L.-J. Li, K. P. Loh, and H. Zhang, Nat. Chem. 5, 263 (2013).

${ }^{11}$ M. Xu, T. Liang, M. Shi, and H. Chen, Chem. Rev. 113, 3766 (2013).

${ }^{12}$ A. K. Geim and I. V. Grigorieva, Nature (London) 499, 419 (2013).

${ }^{13}$ D. Xiao, G. B. Liu, W. Feng, X. Xu, and W. Yao, Phys. Rev. Lett. 108, 196802 (2012).

${ }^{14}$ T. Cao et al., Nat. Commun. 3, 887 (2012).

${ }^{15}$ H. Zeng, J. Dai, W. Yao, D. Xiao, and X. Cui, Nat. Nano. 3, 490 (2012).

${ }^{16}$ K. F. Mak, K. He, J. Shan, and T. F. Heinz, Nat. Nanotechnol. 7, 494 (2012).

${ }^{17}$ G. Sallen, L. Bouet, X. Marie, G. Wang, C. R. Zhu, W. P. Han, Y. Lu, P. H. Tan, T. Amand, B. L. Liu, and B. Urbaszek, Phys. Rev. B 86, 081301(R) (2012).

${ }^{18}$ H. Zeng, Gui-Bin Liu, J. Dai, Y. Yan, B. Zhu, R. He, L. Xie, S. Xu, X. Chen, W. Yao, and X. Cui, Sci. Rep. 3, 1608 (2013).

${ }^{19}$ A. Kormányos, V. Zólyomi, Neil D. Drummond, P. Rakyta, G. Burkard, and Vladimir I. Fal'ko, Phys. Rev. B 88, 045416 (2013).

${ }^{20}$ K. Kośmider and J. Fernández-Rossier, Phys. Rev. B 87, 075451 (2013)

${ }^{21}$ T. Cheiwchanchamnangij and W. R. L. Lambrecht, Phys. Rev. B 85, 205302 (2012).

${ }^{22}$ E. S. Kadantseva and P. Hawrylak, Solid State Commun. 152, 909 (2012).

${ }^{23}$ Z. Y. Zhu, Y. C. Cheng, and U. Schwingenschlögl, Phys. Rev. B 84, 153402 (2011).

${ }^{24}$ H. Ochoa and R. Roldán, Phys. Rev. B 87, 245421 (2013).

${ }^{25}$ W. Kohn and L. J. Sham, Phys. Rev. 140, A1133 (1965).

${ }^{26}$ A. Ramasubramaniam, D. Naveh, and E. Towe, Phys. Rev. B 84, 205325 (2011).

${ }^{27}$ A. Ramasubramaniam, Phys. Rev. B 86, 115409 (2012).

${ }^{28}$ L. Britnell, R. M. Ribeiro, A. Eckmann, R. Jalil, B. D. Belle, A. Mishchenko, Y. J. Kim, R. V. Gorbachev, T. Georgiou, S. V. Morozov, A. N. Grigorenko, A. K. Geim, C. Casiraghi, A. H. Castro Neto, and K. S. Novoselov, Science 340, 1311 (2013).

${ }^{29}$ A. Molina-Sánchez, D. Sangalli, K. Hummer, and A. Marini, and L. Wirtz, Phys. Rev. B 88, 045412 (2013).

${ }^{30}$ N. Marzari, A. A. Mostofi, J. R. Yates, I. Souza, and D. Vanderbilt, Rev. Mod. Phys. 84, 1419 (2012).

${ }^{31}$ H. Shi, H. Pan, Y. W. Zhang, and B. I. Yakobson, Phys. Rev. B 87, 155304 (2013).

${ }^{32}$ C. Espejo, T. Rangel, A. H. Romero, X. Gonze, and G. M. Rignanese, Phys. Rev. B 87, 245114 (2013).
${ }^{33}$ W. Zhang, R. Yu. H.-J. Zhang, X. Dai, and Z. Fang, New J. Phys. 12, 065013 (2010).

${ }^{34}$ H. Min, J. E. Hill, N. A. Sinitsyn, B. R. Sahu, L. Kleinman, and A. H. MacDonald, Phys. Rev. B 74, 165310 (2006).

${ }^{35}$ D. Huertas-Hernando, F. Guinea, and A. Brataas, Phys. Rev. B 74, 155426 (2006).

${ }^{36}$ D. Huertas-Hernando, F. Guinea, and A. Brataas, Phys. Rev. Lett. 103, 146801 (2009).

${ }^{37}$ A. H. Castro Neto and F. Guinea, Phys. Rev. Lett. 103, 026804 (2009).

${ }^{38}$ S. Konschuh, M. Gmitra, and J. Fabian, Phys. Rev. B 82, 245412 (2010).

${ }^{39}$ D. Gosálbez-Martínez, J. J. Palacios, and J. Fernández-Rossier, Phys. Rev. B 83, 115436 (2011).

${ }^{40}$ S. Fratini, D. Gosálbez-Martínez, P. Merodio Camara, and J. Fernández-Rossier, Phys. Rev. B 88, 115426 (2013).

${ }^{41}$ G. Kresse and J. Furthmüller, Phy. Rev. B 54, 11169 (1996).

${ }^{42}$ P. E. Blöchl, Phys. Rev. B 50, 17953 (1994).

${ }^{43}$ G. Kresse and D. Joubert, Phys. Rev. B 59, 1758 (1999).

${ }^{44}$ J. P. Perdew, K. Burke, and M. Ernzerhof, Phys. Rev. Lett. 77, 3865 (1996).

${ }^{45}$ H. J. Monkhorst and J. D. Pack, Phys. Rev. B 13, 5188 (1976).

${ }^{46}$ D. Hobbs, G. Kresse, and J. Hafner, Phys. Rev. B 62, 11556 (2000).

${ }^{47}$ Y.-S. Kim, K. Hummer, and G. Kresse, Phys. Rev. B 80, 035203 (2009).

${ }^{48}$ G. H. Wannier, Phys. Rev 52, 191 (1939).

${ }^{49}$ A. A. Mostofi, J. R. Yates, Y.-S. Lee, I. Souza, D. Vanderbilt, and N. Marzari, Comput. Phys. Commun. 178, 685 (2008).

${ }^{50}$ W. Feng, Y. Yao, W. Zhu, J. Zhou, W. Yao, and D. Xiao, Phys. Rev. B 86, 165108 (2012).

${ }^{51}$ A. Kokalj, Comp. Mater. Sci. 28, 155 (2003).

${ }^{52}$ K. Wittel and R. Manne, Theor. Chim. Acta 33, 347 (1974).

${ }^{53}$ T. M. Dunn, Trans. Faraday Soc. 57, 1441 (1961).

${ }^{54}$ Gui-Bin Liu, Wen-Yu Shan, Yugui Yao, Wang Yao, and Di Xiao, Phys. Rev. B 88, 085433 (2013).

${ }^{55}$ J. C. Slater and G. F. Koster, Phys. Rev. 94, 1498 (1954).

${ }^{56}$ E. Cappelluti, R. Roldán, J. A. Silva-Guillen, P. Ordejón, and F. Guinea, Phys. Rev. B 88, 075409 (2013).

${ }^{57}$ H. Rostami, A. G. Moghaddam, and R. Asgari, Phys. Rev. B 88, 085440 (2013).

${ }^{58}$ G. A. Steele, F. Pei, E. A. Laird, J. M. Jol, H. B. Meerwaldt, and L. P. Kouwenhoven, Nat. Commun. 4, 1573 (2013).

${ }^{59}$ J. Klinovaja and Daniel Loss, Phys. Rev. B 88, 075404 (2013).

${ }^{60}$ X. Li, F. Zhang, and Q. Niu, Phys. Rev. Lett. 110, 066803 (2013).

${ }^{61}$ Y. Song and H. Dery, Phys. Rev. Lett. 111, 026601 (2013).

${ }^{62}$ A. M. Jones, H. Yu, N. J. Ghimire, S. Wu, G. Aivazian, J. S. Ross, B. Zhao, J. Yan, D. G. Mandrus, D. Xiao, W. Yao, and X. Xu, Nat. Nanotechnol. 8, 634 (2013).

${ }^{63}$ F. Parhizgar, H. Rostami, and R. Asgari, Phys. Rev. B 87, 125401 (2013).

${ }^{64}$ Wen-Yu Shan, Hai-Zhou Lu, and Di Xiao, Phys. Rev. B 88, 125301 (2013).

${ }^{65}$ A. Kormányos, V. Zólyomi, N. D. Drummond, and G. Burkard, arXiv:1310.7720. 